\title{
An Evaluation of EEG Scanner's Dependence on the Imaging Technique, Forward Model Computation Method, and Array Dimensionality
}

Stahlhut, Carsten; Attias, Hagai Thomas ; Stopczynski, Arkadiusz; Petersen, Michael Kai; Larsen, Jakob Eg; Hansen, Lars Kai

Published in:

2012 Annual International Conference of the IEEE Engineering in Medicine and Biology Society (EMBC)

Link to article, DOI:

10.1109/EMBC.2012.6346235

Publication date:

2012

Link back to DTU Orbit

Citation $(A P A)$ :

Stahlhut, C., Attias, H. T., Stopczynski, A., Petersen, M. K., Larsen, J. E., \& Hansen, L. K. (2012). An Evaluation of EEG Scanner's Dependence on the Imaging Technique, Forward Model Computation Method, and Array Dimensionality. In 2012 Annual International Conference of the IEEE Engineering in Medicine and Biology Society (EMBC) (pp. 1538-1541). IEEE. I E E E Engineering in Medicine and Biology Society. Conference Proceedings https://doi.org/10.1109/EMBC.2012.6346235

\section{General rights}

Copyright and moral rights for the publications made accessible in the public portal are retained by the authors and/or other copyright owners and it is a condition of accessing publications that users recognise and abide by the legal requirements associated with these rights.

- Users may download and print one copy of any publication from the public portal for the purpose of private study or research.

- You may not further distribute the material or use it for any profit-making activity or commercial gain

- You may freely distribute the URL identifying the publication in the public portal 


\title{
An Evaluation of EEG Scanner's Dependence on the Imaging Technique, Forward Model Computation Method, and Array Dimensionality
}

\author{
Carsten Stahlhut ${ }^{1}$, Hagai Thomas Attias ${ }^{2}$, Arkadiusz Stopczynski ${ }^{1}$, \\ Michael Kai Petersen ${ }^{1}$ Jakob Eg Larsen ${ }^{1}$, Lars Kai Hansen ${ }^{1}$.
}

\begin{abstract}
EEG source reconstruction involves solving an inverse problem that is highly ill-posed and dependent on a generally fixed forward propagation model. In this contribution we compare a low and high density EEG setup's dependence on correct forward modeling. Specifically, we examine how different forward models affect the source estimates obtained using four inverse solvers Minimum-Norm, LORETA, MinimumVariance Adaptive Beamformer, and Sparse Bayesian Learning.
\end{abstract}

\section{INTRODUCTION}

Electro-encephalography (EEG) holds great promise for functional brain imaging due to its high temporal resolution. In comparison with functional magnetic resonance imaging (fMRI) and positron emission tomography (PET), the slow hemodynamic response does not affect EEG. In addition fMRI and PET involve heavy scanner equipment and immobilization constraints that compromise the experimental situation, while EEG can be performed under much more natural conditions.

Today EEG based brain imaging suffers from a lack of spatial specificity due to the complex propagation of neural quasi-static electric fields to the array of sensors placed at the scalp surface. Motivated by the desire to perform reliable and precise reconstruction of the neural current density, much effort has been devoted to development of improved inversion methods. The current literature can be divided in two major approaches: Equivalent current dipole (ECD) and distributed models. In ECD methods [1] it is assumed that the brain activity is generated by a small number of focal sources, which restricts the source localization problem to a challenge of determining the positions and orientations of the ECDs. In distributed models several prior assumptions are made in order to solve the ill-posed inverse problem. For example $l_{2}$-norm approaches, like the weighted minimum norm method [2] and low resolution electromagnetic tomography (LORETA) [3], assume sources to be diffuse and highly distributed. On the other hand models based on the $l_{1}$-norm [4], $l_{p}$-norms [5], minimum variance beamformer [6], Bayesian model averaging [7], multiple priors models [8], and automatic relevance determination methods [9], [10], implement more focal sources. Most of these source

\footnotetext{
*This work is supported in part by the Danish Lundbeck Foundation through CIMBI Center for Integrated Molecular Brain Imaging.

${ }^{1}$ DTU Informatics, Technical University of Denmark, DK-2800 Kgs. Lyngby. C. Stahlhut : CS at imm.dtu.dk

${ }^{2}$ Convex Imaging, San Francisco, CA 94108, USA
}

localization methods employ spatial-temporal priors in order to accommodate for the focal source distribution.

While the recent EEG imaging literature mainly have focused on the source reconstruction performance using high density EEG equipment we here draw the attention to quantify the performance of EEG brain imaging using few electrodes as we are interested in mobile EEG equipment. We have previously, demonstrated the feasibility of performing online brain imaging on a smartphone device [11] allowing for experiments in more naturalistic settings. We here seek a quantification of how well the current sources can be reconstructed when evaluating different forward models and source reconstruction methods.

Analyzing the importance of precise forward models and their influence on either the forward problem or the inverse problem is far from new to the EEG community. A number of contributions have already been published e.g. [12], [13]. A majority of the forward model investigations performed evaluates the forward models at sensor level or only examine a few dipoles located in different brain regions. These contributions have been of crucial importance to the EEG community as they have shed light on serious issues that we need to be aware of when the source solutions are used as the basis of conclusions in a given setup. In [12] an examination of the influence of geometric errors on the source estimates is performed using BEM models constructed from MR or CT images. A quite similar approach as the analysis above have been presented in [14]. The study in [14] deals with geometric errors introduced by using too simple head models (spheres) compared to more realistic BEM models. To evaluate the effect, angles between forward fields were examined when assuming no noise at the sensor level. In contrast to [14] we explored forward model uncertainties in face of noise present at the sensor level [15] and demonstrated that indeed source confusion is dependent on the interplay of forward field errors and the amount of noise present in the recordings.

Other types of uncertainities affecting the forward models are the specific tissue conductivity values and the importance of modeling specific tissues as anisotropic rather than isotropic have been discussed in [13], with the overall consensus that inaccurate modeling of the skull leads to significant error contributions on the sources. In fact [13], [16] states that a smearing effect on the forward potential computation is introduced by the skull anisotropy. The deeper 
a source is located the more it is surrounded by anisotopic tissues. Thus, electric fields generated by deeper sources are more affected by the anisotropy than superficial sources.

Of more recent studies [17] should be mentioned in which a careful analysis of how the number of electrodes, geometric errors (spheres versus FEM head models), and anisotropy versus isotropy affect the source estimates obtained by beamformers. To the authors knowledge this is the first study comparing how different inverse methods solutions are affected by different choices of forward models as well as the resolution with respect to the number sensors.

\section{METHODS}

The relation between the measured EEG signal and the brain's current sources can be expressed as a linear instantaneous form in the sources. The forward relation can be written as [18]

$$
\mathbf{Y}=\mathbf{A X}+\mathcal{E}
$$

where the measured EEG signal is denoted $\mathbf{Y} \in \Re^{N_{c} \times N_{t}}$, the current sources $\mathbf{X} \in \Re^{N_{d} \times N_{t}}$, and the noise $\mathcal{E}$ is assumed additive. Number of channels, dipoles (or sources), and time samples are denoted $N_{c}, N_{d}$, and $N_{t}$, respectively. The coupling of sensors and the current sources is expressed through the lead field matrix/forward model $\mathbf{A} \in \Re^{N_{c} \times N_{d}}$ with the rows referred to as the lead fields for the sensors and the columns as the forward fields for the sources. The forward model depends on sensor positions, a so-called 'head model' of the spatial distribution of tissue, and tissue conductivity values. Multiple methods based on the physical properties of the brain and Maxwell's equations are available for computing $\mathbf{A}$.

\section{A. Minimum Norm and LORETA}

Given the linear relation in Eq. (1) and if we assume the noise to be time independent Gaussian distributed, the observation model becomes $p(\mathbf{Y} \mid \mathbf{X})=$ $\prod_{t=1}^{N_{t}} \mathcal{N}\left(\mathbf{y}_{t} \mid \mathbf{A} \mathbf{x}_{t}, \beta^{-1} \boldsymbol{\Sigma}_{\mathcal{E}}\right)$ where $\boldsymbol{\Sigma}_{\mathcal{E}}$ is the noise spatial covariance matrix. We here realize the source localization by a Bayesian formulation of the widely used minimum norm (MN) [2] and LORETA [3] methods as they allow fast computation of the inverse solution. With $\mathrm{MN}$ regarded as a special case of the LORETA we use as prior distribution for the sources a multivariate Gaussian $p(\mathbf{X} \mid \alpha)=$ $\prod_{t=1}^{N_{t}} \mathcal{N}\left(\mathbf{x}_{t} \mid \mathbf{0}, \alpha^{-1} \mathbf{L}^{T} \mathbf{L}\right)$ with $\mathbf{L}$ being the Laplacian operator incorporating spatial smoothness on the source level. $\mathrm{MN}$ is obtained in the limit of no spatial smoothness, i.e. replacing $\mathbf{L}=\mathbf{I}$. Source estimates are now obtained from Bayes' rule by computing the posterior distribution over the sources, which leads to $p(\mathbf{X} \mid \mathbf{Y})=\prod_{t=1}^{N_{t}} \mathcal{N}\left(\mathbf{x}_{t} \mid \boldsymbol{\mu}_{t}, \boldsymbol{\Sigma}_{x}\right)$ with

$$
\begin{aligned}
\boldsymbol{\Sigma}_{x} & =\alpha^{-1} \mathbf{K}-\alpha^{-1} \mathbf{K} \mathbf{A}^{T} \boldsymbol{\Sigma}_{y} \mathbf{A} \mathbf{K} \alpha^{-1} \\
\boldsymbol{\mu}_{t} & =\alpha^{-1} \mathbf{K A}^{T} \boldsymbol{\Sigma}_{y} \mathbf{y}_{t} .
\end{aligned}
$$

Here we have defined $\mathbf{K} \equiv \mathbf{L}^{T} \mathbf{L}$ and $\boldsymbol{\Sigma}_{y}^{-1} \equiv$ $\alpha^{-1} \mathbf{A} \mathbf{K A}^{T}+\beta^{-1} \boldsymbol{\Sigma}_{\varepsilon}$. Estimation of sources and the precision parameters $\alpha$ and $\beta$ are carried out using a standard expectation-maximization (EM) scheme [19].

\section{B. Beamforming}

Here we use minimum-variance adaptive beamforming, which reconstructs the signal $s_{i}$ of each dipole $i$ by a spatial filter, $s_{i}=\sum_{j} W_{i j} y_{j}$. It chooses the filter that minimizes the noise variance in the reconstructed signal under a unit-gain constraint. Thus, for each $i$, we seek

$$
\hat{W}_{i j}=\operatorname{argmin}_{\mathbf{W}}\left(\mathbf{W} \boldsymbol{\Sigma}_{\mathcal{E}} \mathbf{W}^{T}\right)_{i i}, \quad(\hat{\mathbf{W}} \mathbf{A})_{i i}=1 .
$$

The resulting reconstruction is given by

$$
x_{i}=\frac{\left(\mathbf{A}^{T} \mathbf{C}_{y}^{-1} y\right)_{i}}{\left(\mathbf{A}^{T} \mathbf{C}_{y}^{-1} \mathbf{A}\right)_{i i}}
$$

where $\mathbf{C}_{y}$ is the empirical data covariance. In dense EEG systems, where the number of sensors exceeds 100, this covariance is low-rank and must be suitably regularized. However, in systems with a small number of sensors, such as the one described here, regularization is usually not required.

\section{Sparse Bayesian Learning (SBL)}

SBL is a promising recent addition to the source analysis toolkit. In constrast to the previous methods which result in brain images with a rather low spatial resolution, SBL's images are very sparse. This sparseness is achieved by modeling each dipole distribution by a Gaussian with its own separate precision parameter $\alpha_{i}, p(\mathbf{X})=\prod_{t=1}^{N_{t}} \mathcal{N}\left(\mathbf{x}_{t} \mid 0, \mathbf{D}^{-1}\right)$, where $\mathbf{D}=\operatorname{diag}(\boldsymbol{\alpha})$ leading to a posterior distribution

$$
\begin{aligned}
p(\mathbf{X} \mid \mathbf{Y})=\prod_{t=1}^{N_{t}} \mathcal{N}\left(\mathbf{x}_{t} \mid \boldsymbol{\mu}_{t}, \boldsymbol{\Sigma}_{x}\right) \text { with } \\
\boldsymbol{\Sigma}_{x}^{-1}=\mathbf{A}^{T} \beta \boldsymbol{\Sigma}_{\mathcal{E}}^{-1} \mathbf{A}+\mathbf{D} \\
\boldsymbol{\mu}_{t}=\boldsymbol{\Sigma}_{x} \mathbf{A}^{T} \beta \boldsymbol{\Sigma}_{\mathcal{E}}^{-1} \mathbf{y}_{t} .
\end{aligned}
$$

The precision parameters are estimated from data by a EMlike algorithm, made efficient using a convex optimization technique. The update rule for $\boldsymbol{\alpha}$ is given in [9].

\section{EMPIRICAL EVALUATION}

We demonstrate the influence of the choices of forward models on the source estimates depending on which inverse method that is used. In order to validate how the inverse methods are affected by these choices we focus on two different EEG setups, Emotiv EPOC (16 channels) and Biosemi Active-II system (64 channels). For each of the EEG setups we examine three types of head models; 3-spheres (SPM8 toolbox), BEM-CP (SPM8 toolbox) [20], BEM-OP (OpenMEEG toolbox) [21] all using the same cortical surface with a resolution of 5,124 vertices. In contrast to spheres models BEM models face high numerical challenges in order to extract the forward fields at the cortical level. A limitation with the BEM-CP implementation is a risk of improper handling of forward fields for vertices very close to the inner skull. This issue is illustrated in Fig. 1 in which we demonstrate the 2-norm of each of the 5,124 vertices forward fields. We note that the BEM-CP has a number of vertices having forward fields much larger than the average. In this section we inspect how such discontinuties in the electric field may affect the source solutions. 


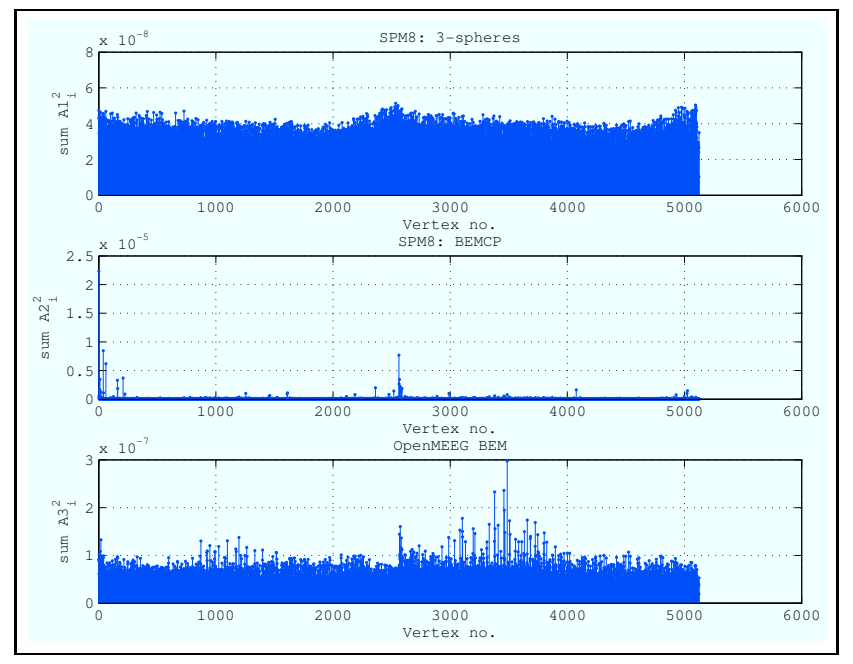

Fig. 1. The distribution of the squared 2-norm of the forward fields for three different forward models 3-spheres, BEM-CP, and BEM-OP when using 64-channel Biosemi layout.

Evaluation is carried out on synthetic data. We select a small cortical area in the right temporal lope as being active and with a half sine as temporal signature. Fig.2.a shows the spatial distribution. As true forward propagation model we apply the OpenMEEG BEM model with tissue conductivities brain:skull:scalp $=0.33: 0.0041: 0.33 \mathrm{~S} / \mathrm{m}$. Gaussian noise is added to the clean EEG signal in the order of $\mathrm{SNR}=10$, see Fig.2.b. We define SNR as the ratio between the power of the clean EEG and the noise. Fig.2.c shows the source solutions of MN, LORETA, MVAB, and SBL methods when applied to a low resolution setup of 16 channels (Emotiv) and high density 64-channels (Biosemi). Overall MN, LORETA, and MVAB all leads to widespread activity with MVAB having difficulties to capture the simulated source in the temporal lope. MN seems to be more affected by the BEMCP's discontinuties in the electric field for the Emotiv setup compared to Biosemi. However, LORETA minimizes the influence of these discontinuties by its spatial smoothness. The source estimates of the SBL algorithm on the other hand leads to highly sparse solutions with a few strong sources located close to the true source region.

\section{CONCLUSIONS}

We examined inaccurate forward models influence on the source reconstruction in a low and high density EEG setup. Source solutions obtained using MN, LORETA, and SBL demonstrated possibility of recovering sources located in the temporal lope at a $\mathrm{SNR}=10$ reliable for most of the forward models and with SBL being the method resulting in the most consisting source estimates for the the different forward models. Further studies should evaluate the performance under lower SNRs and with multiple source regions being active simultaneously. Moreover, forward models considering anisotropy such as Finite Volume Model and Finite Element Model would be interested to include in the comparison.

\section{REFERENCES}

[1] M. Scherg, T. Bast, and P. Berg, "Multiple source analysis of interictal spikes: Goals, requirements, and clinical value," J. Clin. Neurophysiol., vol. 16, pp. 214-224, 1999.

[2] M.S. Hämäläinen and R.J. Ilmoniemi, "Interpreting magnetic fields of the brain: minimum norm estimates," Med. Biol. Eng. Comput., vol. 32, pp. 35-42, 1994.

[3] R.D. Pascual-Marqui, M. Michel, and D. Lehmann, "Low resolution electromagnetic tomography: A new method for localizing electrical activity in the brain," Int J. Psychophys, vol. 18, pp. 49-65, 1994.

[4] M. Fuchs, M. Wagner, T. Kohler, and H.A. Wischman, "Linear and nonlinear current density reconstructions," J. Clin. Neurophysiol., vol. 16, no. 3, pp. 267-295, 1999.

[5] T. Auranen, A. Nummenmaa, M. Hämäläinen, I. Jääskeläinen, J. Lampinen, A. Vehtari, and M. Sams, "Bayesian analysis of the neuromagnetic inverse problem with $\ell^{p}$-norm priors," Neurolmage, vol. 26, pp. 870-884, 2005.

[6] F. Darvas, D. Pantazis, E. Kucukaltun Yildrium, and R. Leahy, "Mapping human brain function with MEG and EEG: methods and validation," NeuroImage, vol. 25, pp. 383-394, 2004.

[7] N.J. Trujillo-Barreto, E. Aubert-Vazquez, and P.A. Valdes-Sosa, "Bayesian model averaging in EEG/MEG imaging," NeuroImage, vol. 21, no. 4, pp. 1300-1319, 2004.

[8] K. Friston, L. Harrison, J. Daunizeau, S. Kiebel, C. Phillips, N. Trujillo-Barreto, R. Henson, G. Flandin, and J. Mattout, "Multiple sparse priors for the M/EEG inverse problem," NeuroImage, vol. 39, pp. 1104-1120, 2008.

[9] D. P. Wipf, J. P. Owen, H. T. Attias, K. Sekihara, and S. S. Nagarajan, "Robust Bayesian estimation of the location, orientation, and time course of multiple correlated neural sources using MEG," Neuroimage, vol. 49, no. 1, pp. 641-655, 2010.

[10] Carsten Stahlhut, Hagai T. Attias, David Wipf, Lars K. Hansen, and Srikantan S. Nagarajan, "Sparse spatio-temporal inference of electromagnetic brain sources," in Machine Learning in Medical Imaging, vol. 6357 of Lecture Notes in Computer Science, pp. 157164. Springer Berlin / Heidelberg, 2010.

[11] A. Stopczynski, J. Larsen, C. Stahlhut, M. Petersen, and L. Hansen, "A smartphone interface for a wireless eeg headset with real-time $3 \mathrm{~d}$ reconstruction," Affective Computing and Intelligent Interaction, pp. 317-318, 2011.

[12] G. Huiskamp, M. Vroeijenstijn, R. van Dijk, G. Wieneke, and A.C. van Huffelen, "The need for correct realistic geometry in the inverse EEG problem," IEEE Trans. Biomed. Eng., vol. 46, no. 11, pp. 12811287, Nowember 1999.

[13] C.H. Wolters, A. Anwander, X. Tricoche, D. Weinstein, M.A. Koch, and R.S. MacLeod, "Influence of tissue conductivity anisotropy on EEG/MEG field and return current computation in a realistic head model: A simulation and visualization study using high-resolution finite element modeling," Neuroimage, vol. 30, no. 3, pp. 813-826, 2006.

[14] M. Fuchs, R. Drenckhahn, H. Wischmann, and M. Wagner, "An improved boundary element method for realistic volume-conductor modeling," IEEE Transactions on Biomedical Engineering, vol. 45, no. 8, pp. 980-997, 1998.

[15] Carsten Stahlhut, Functional Brain Imaging by EEG: A Window to the Human Mind, Ph.D. thesis, Technical Univeristy of Denmark, DTU Informatics, DK-2800 Richard Petersens Plads, Denmark, 2011.

[16] H. Hallez, B. Vanrumste, R. Grech, J. Muscat, W. De Clercq, A. Vergult, Y. D'Asseler, K.P. Camilleri, S.G. Fabri, S. Van Huffel, et al., "Review on solving the forward problem in EEG source analysis," $J$ of NeuroEng. and Rehab, vol. 4, no. 1, pp. 46, 2007.

[17] O. Steinsträter, S. Sillekens, M. Junghoefer, M. Burger, and C.H. Wolters, "Sensitivity of beamformer source analysis to deficiencies in forward modeling," Human brain mapping, vol. 31, no. 12, pp. 1907-1927, 2010.

[18] S. Baillet and L. Garnero, "A bayesian approach to introducing anatomo-functional priors in the EEG/MEG inverse problem," IEEE Trans. on Biomedical Engineering, vol. 44, no. 5, pp. 374-385, 1997.

[19] C. M. Bishop, Pattern Recognition and Machine Learning, Springer, NY 10013 (USA), 2006.

[20] C.L.M. Phillips, "Source estimation in EEG: Combining anatomical and functional constraints," PhD Thesis, 2000.

[21] A. Gramfort, T. Papadopoulo, E. Olivi, M. Clerc, et al., "Openmeeg: opensource software for quasistatic bioelectromagnetics," Biomedical engineering online, vol. 9, no. 1, pp. 45, 2010. 


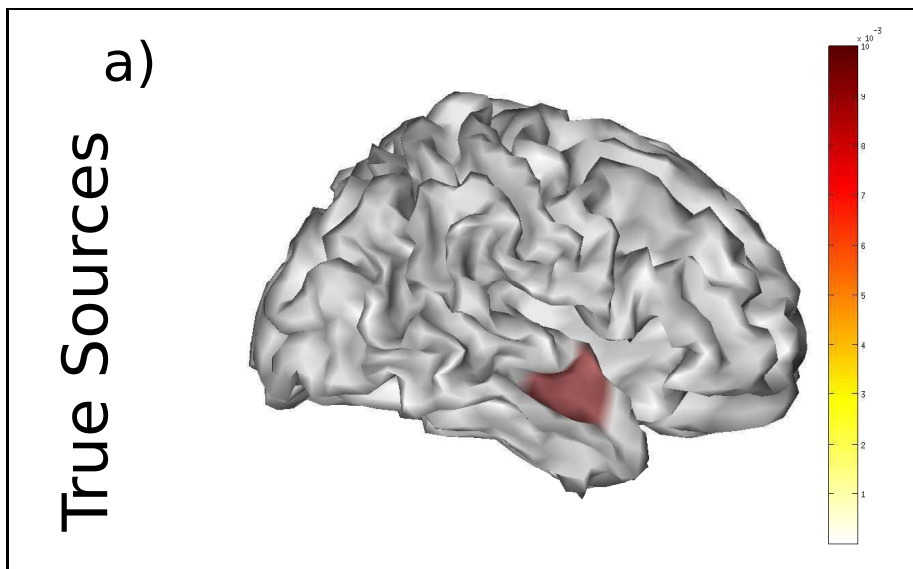

b)

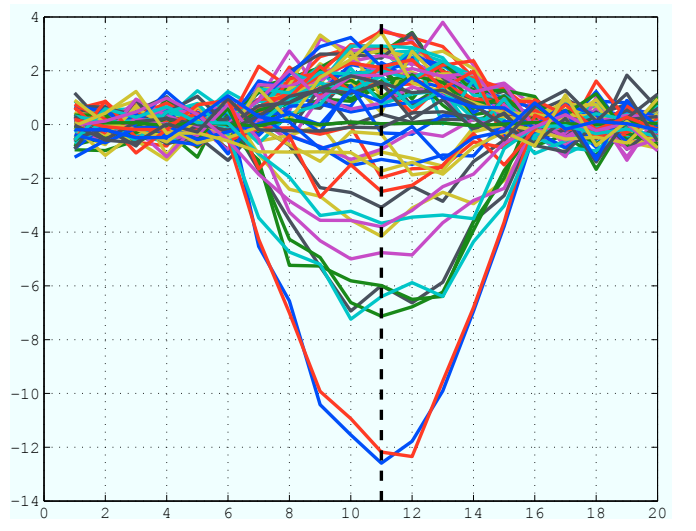

c)

LORETA

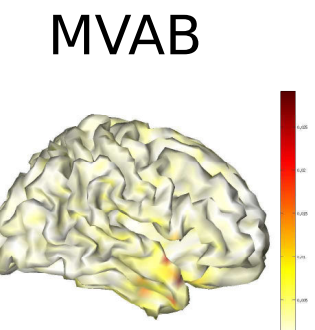

SBL
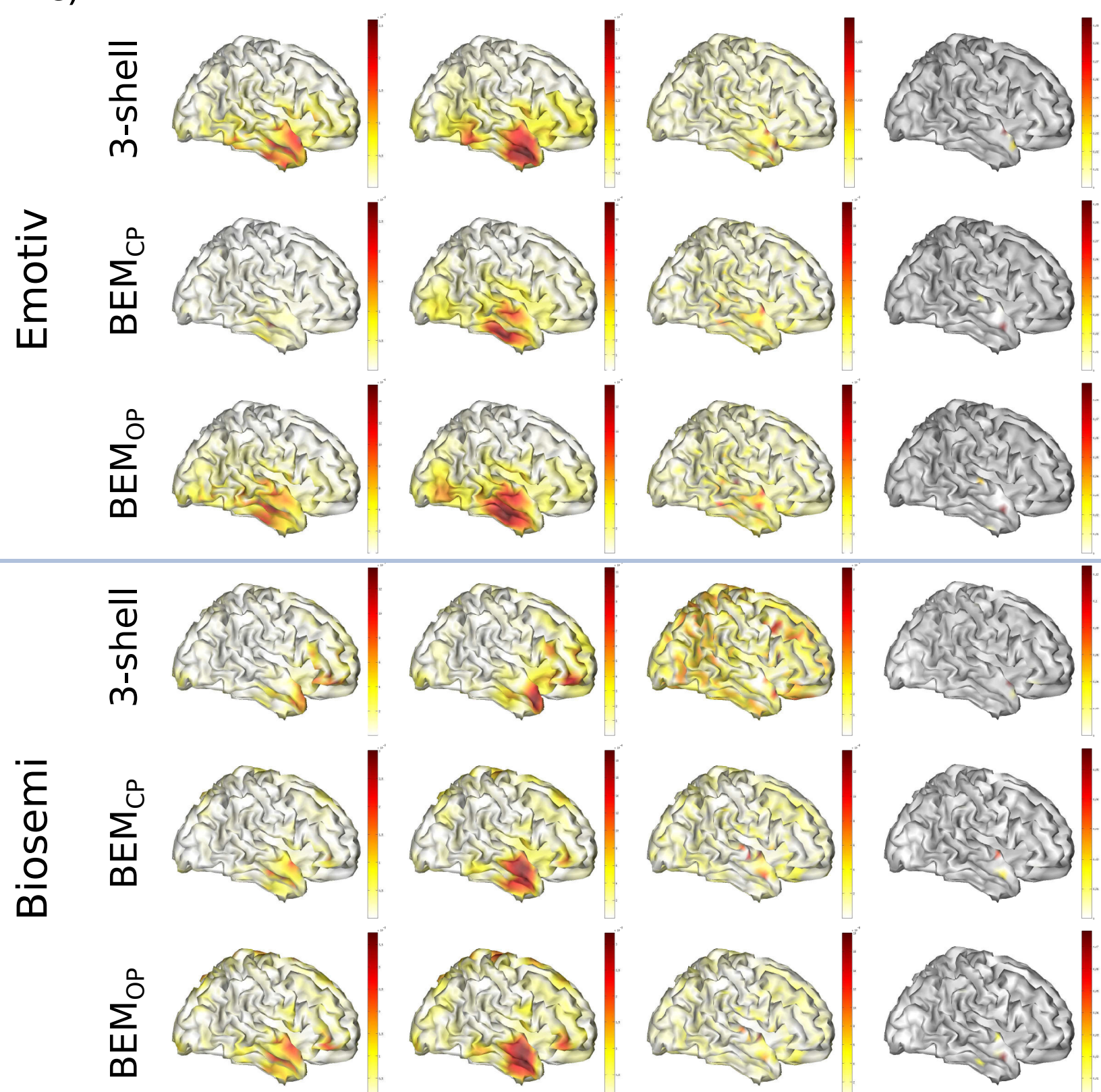

Fig. 2. a) Simulated spatial source distribution. b) Simulated sensor signal including noise. c) Reconstructed source estimates using MN, LORETA, MVAB, and SBL on two different EEG setups, Emotiv $\left(N_{c}=16\right)$ and Biosemi $\left(N_{c}=64\right)$ and three head models: 3 -shell, BEM-CP, and BEM-OP. 